This item was submitted to Loughborough's Research Repository by the author.

Items in Figshare are protected by copyright, with all rights reserved, unless otherwise indicated.

\title{
Improving reuse of in-service information capture and feedback
}

PLEASE CITE THE PUBLISHED VERSION

http://dx.doi.org/10.1108/17410380910961028

PUBLISHER

(C) Emerald

VERSION

AM (Accepted Manuscript)

LICENCE

CC BY-NC-ND 4.0

REPOSITORY RECORD

Goh, Yee M., and Chris McMahon. 2019. "Improving Reuse of In-service Information Capture and Feedback". figshare. https://hdl.handle.net/2134/8078. 
This item was submitted to Loughborough's Institutional Repository (https://dspace.lboro.ac.uk/) by the author and is made available under the following Creative Commons Licence conditions.

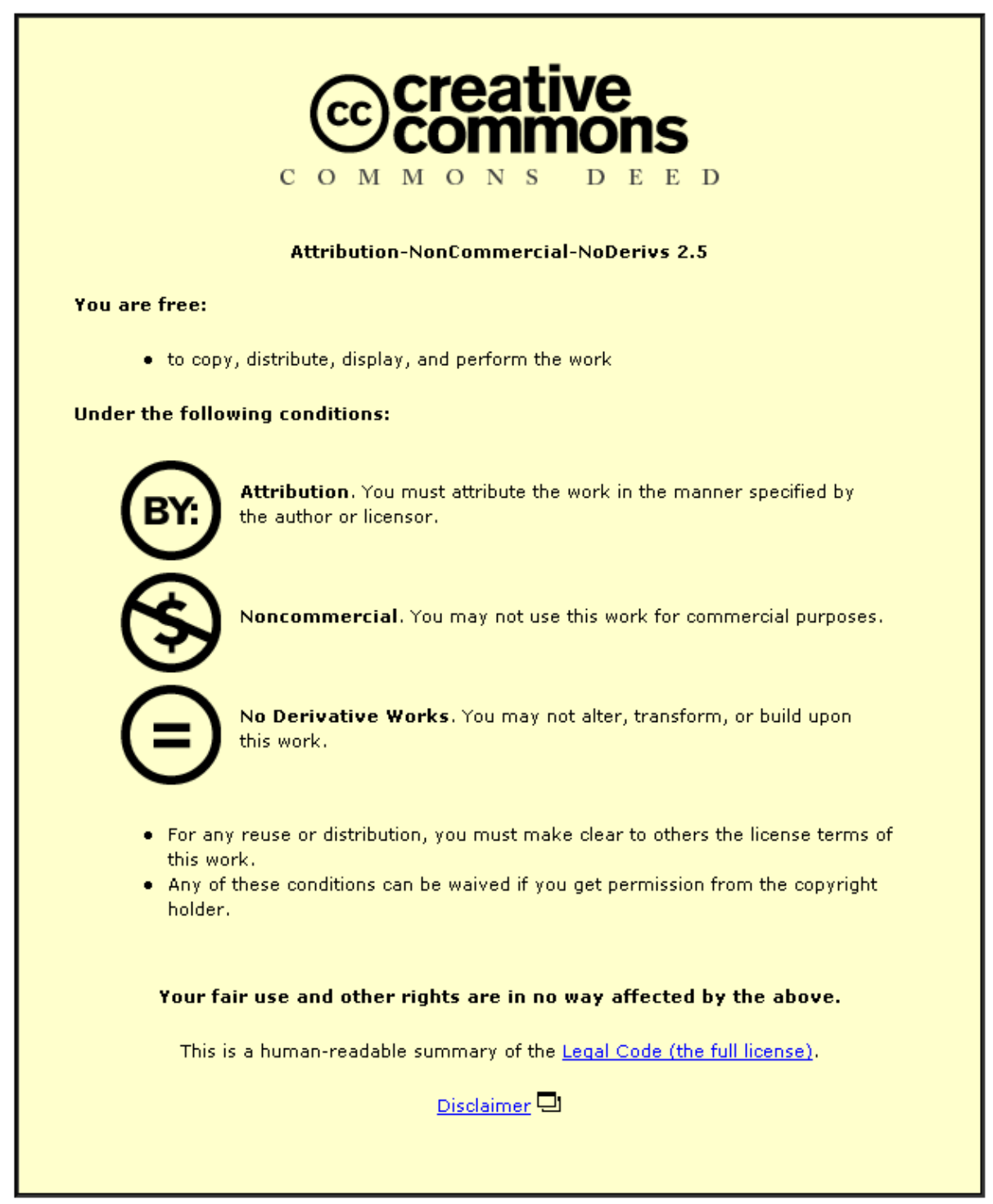

For the full text of this licence, please go to: http://creativecommons.org/licenses/by-nc-nd/2.5/ 


\title{
Improving reuse of in-service information capture and feedback
}

\author{
Yee Mey Goh", Chris McMahon \\ Department of Mechanical Engineering, University of Bath, UK
}

The adoption of the PSS business model means that the designing company has greater scope and motivation to learn from experience of its products in use to improve their core design and engineering capabilities. Continuous improvement, however, depends crucially upon the implementation of effective Knowledge and Information Management (KIM) systems within a dynamic learning environment that impinge on diverse communities throughout the product lifecycle. This paper consolidates literature reviews and presents our empirical observations relating to the current KIM systems and practices within large aerospace and manufacturing companies. In particular, we discuss issues and experiences from a case study to enhance reuse of in-service feedback conducted with an aerospace company. The case study involves the organisation of inservice records to expedite retrieval and knowledge discovery.

Keywords: Service feedback, codification, information reuse, learning from experience, Product-Service System

\section{Introduction}

A Product-Service System (PSS) is an integrated solution that fulfils functions and provides services to endusers without necessarily transferring the ownership of the product to them. This is known to increase longterm business revenue and stability to the supplying or designing companies, whilst in turn creating benefits to customers with guaranteed availability and lower capital costs (Alonso-Rasgado et al. 2004). A number of high profile companies have been quoted as having adopted the PSS model in diverse applications, including Xerox, Canon, IBM and Rolls-Royce. In fact, the actual composition of the product and service of a PSS spans the continuum between pure products at one end and pure services at the other. Notionally, three types of PSS solutions have been identified (Baines, 2007), namely the product-oriented, use-oriented and resultoriented type, each with different emphasis to deliver the required function. In a product-oriented type PSS, the traditional product offering companies are offering more and more service packages comprising of installation, maintenance contracts, overhaul and repair, upgrades and training. This is seen as a win-win situation as customers can have less personnel involvement in maintenance, at the same time, companies can expand its business opportunity.

In the context of the PSS, products are upgraded, remanufactured and used for different purposes through their extended life. Central to the shift towards PSS businesses is that the designing company has greater scope and motivation to learn from its involvement in the in-service activities. This involvement means that in-service information and knowledge become more accessible to the designing company (Ward and Graves 2006) in contrast to the traditional model where products were designed and manufactured but often, maintained and serviced by different companies. Because the PSS is now a single system, there is also greater incentivisation structure to continuously learn from operational and in-service activities and to capitalise on

*Corresponding author. Email: y.m.goh@bath.ac.uk Tel: +44(0)1225386131 Fax: +44 (0)1225 386928 
this opportunity to improve the companies' core design and engineering capabilities. Continuous improvement depends crucially upon the implementation of effective Knowledge and Information Management (KIM) systems that can be usefully deployed to enhance engineering decisions throughout the life cycle and beyond. What are the challenges and how should the PSS companies take full advantage of this opportunity to learn from the information captured in service for its continuous improvement?

Companies can use insights gained from use and in-service to adapt their on-going support activities and also to feed-forward this knowledge into new design projects. Therefore, it is important that this knowledge is captured, managed and made available, both from the product and system perspective and from the people perspective. To this end, codification strategies seek to embed knowledge in formal information resources (Hansen et al. 1999). Codification methods capitalise on the maturity of Information Technology (IT) tools for storage, organisation and distribution of information. Equally important but not the focus of this paper, is the personalization approaches to knowledge management. Personalization approaches promote the development and retention of knowledge by individuals and communities (Hansen et al. 1999). Focusing on the codification strategies, the problem relates to how information and knowledge concerning the ongoing aspects of in-service operation such as maintenance requirements, performance and reliability of the product can be captured and reused.

This paper is not about how to engineer the PSS but discusses information reuse that is considered to be an even more important aspect of a PSS business compared to a product offering business. Firstly our empirical observation relating to the feedback processes made within large aerospace and manufacturing companies is compared with literature findings, and then our experiences with in-service information capture and reuse are discussed.

\section{In-service feedback}

In-service feedback to the engineering organisation can be achieved via two fundamentally different means. The personalisation approach is essentially concerned with the development of communities of practice (CoPs) and socio-technical models for enhancing company performance (Wenger 2000). This approach emphasises on the dynamic capabilities of organisations to learn in response to changing circumstances. The second approach, codification, is concerned with making knowledge explicit through its capture and formal representation such that it can be reused. Codification strategies require knowledge gained to be codified into suitable representation, stored and organised such that the information may be used at a later stage. In practice, a combination of personalisation and codification techniques are necessary and complementary. This section provides a review of in-service feedback in literature and reports our empirical observations.

\subsection{Literature review}

In studying the feedback of reliability information into development process of high-volume consumer products, Sander and Brombacher (2000) found that reactive loop between operations and development team does exist. However, they could not identify pro-active learning loops from the interviews conducted in a company. They noted a number of issues related to the culture and organisational structure that do not encourage, and worse still, may impede aspirations of continuous improvement. They suggested improvement to business processes through promoting communication and improving closed-loop information flows between departments. From case studies with three companies, Fundin (2003) also found that none of the companies actually learn from feedback information to inform new product developments. The feedback systems (customer feedback systems and codified and personalised information provided by service personnel) at best were used to improve current product development processes. Busby (1998) conducted both structured and semi-structured interviews on feedback in design and identified problems associated with it. 
Their observations included informational, motivational and organisational factors that make feedback problematic and difficult.

As service contracts demand for ever shorter time for responses from engineering companies, learning from use is important to enhance the ongoing maintenance and service activities. General Motors (GM) OnStar telematics service, for instance, uses a combination of Global Positioning System (GPS) satellite and mobile phone network, along with onboard diagnostics, to link the vehicle and driver to the OnStar centre. The system enables GM to provide quicker repair and maintenance services. This type of learning is equivalent to the single-loop learning which is reactionary and that results in knowledge that is not embodied in the product (Maidique 1985, Argyris 1999). The single-loop learning may be seen in terms of changing maintenance schedules or operating modes, developing workaround solutions etc. in order to avoid recurring in-service issues. This mode of learning is commonly found in industry and application of data monitoring systems is mainly to support this mode. However, more importantly, and arguably more beneficial to the PSS companies, is the double-loop learning that completes the feedback loop, i.e. where learning will affect the design of the product and therefore resulting in embodied knowledge. The double-loop learning is inevitably more difficult to achieve, as the observation is made by service engineers but learning needs to be made by the designers in spite of the issues described in section 2.2. Nonetheless, GM for example, is planning to use the customer insight to better design its future vehicles to meet customer needs (Koudal 2004).

The continuous improvement aspects associated with the PSS has been pointed out by a number of authors. In the context of PSS, a number of literature papers have identified the importance of in-service knowledge to the improvement of design. Doultsinou et al. (2007), from a series of semi-structured interviews conducted in the UK manufacturing industry, identified the types of service knowledge related to tooling, spares, serviceability, maintenance and training. The significance of in-service knowledge was highlighted, more recently, by a Department of Trade and Industry (DTI) funded project on Integrated Product and Service (IPAS). In this context, Jagtap et al. (2007) conducted a series of questionnaire and interviews with designers and service engineers to determine the most important and common life cycle information design engineers seek from maintenance documents. They arrived at categories of information designers find useful, such as information related to component failure, operating conditions, maintenance, life cycle cost and reliability. They also noted that in-service information required by the designers is highly heterogeneous and disparate. Through the IPAS project, (Wong et al. 2007) employed an ontological approach to organise service information to support the ability to make inference between design and in-service outcomes. They have developed a prototype system that allows the users to access previously heterogeneous document resources, integrated by a shared ontology via a web portal. Márquez and Herguedas (2004) analysed the maintenance records for earthmoving equipment in order to determine root causes with the objective to improve the current maintenance processes. Although feasible, they noted a number of challenges such as ineffective maintenance data management and reduced knowledge in data processing techniques. Although opportunities for learning have been recognised in addition to business advantages in the PSS (Tan and McAloone 2006), methods for utilising information and knowledge feedback from the later life cycle phases to impact the PSS activities have been limited in the literature.

\subsection{Empirical observation}

Our empirical observations suggest that the feedback processes are in general ad hoc and informal although formal procedures such as engineering change requests do exist. A combination of personalization and codification approaches are used, but all companies are placing greater emphasis on the development of information systems as they face greater pressure to get design right first time due to outsourcing and global competition. These efforts quite often are faced with resistance from the employees, as they require additional time and efforts that are not counted towards productive work (e.g. designing). There has also been expression 
of insecurity and fear of losing ownership of knowledge if everything is codified. Usually in the same organisation, some departments, work groups or projects tend to be more active at exploiting feedback than others but such experiences and best practices are not shared outside of the groups.

Previously, contracted companies may be providing third party servicing which makes service information and knowledge less visible and in some cases, inaccessible to the designing companies. Even though in the PSS, servicing activities are being carried out by the same company, the functional divisions (e.g. design, production, service), especially in large companies, along with roles and responsibilities frequently acts as information and knowledge barriers, i.e. service engineers will have more knowledge of customers' needs and requirements yet may not necessarily share this understanding with the design engineers. This means that only limited feedback reaches the design teams. There are many reasons for this, including the nature of the service business that is geographically distributed, for instance, teams may be stationed away from their companies' main offices to be closer to the install base. Some products may be installed in remote locations or may not have fixed positions such as on aircraft or ships. Due to the amount of time spent away from the main office, service teams often find it difficult to share knowledge through traditional face-to-face contact. In addition, the main concerns of service engineers are to resolve issues and to bring the systems up and running as soon as possible. Hence there is much reduced priority in producing detailed documentation of their work. In isolated cases, technological and communication difficulties on site may also prevent service engineers from accessing and logging information into company's information systems. As a result of such motivational and technological barriers, feedback from service is found to be less than optimal.

More encouragingly, as companies are involved in providing services and developing long-term strategic relationships with its customers, learning from in-service issues increasingly gained attention in all the companies that we have interacted with. This was evident by the fact that service engineers being invited to design reviews which was previously not the case. Previously, service engineers will send the components back to the design team for diagnosis without passing on information about the operating conditions on-site that may be extremely useful to the designers. It is interesting to note that service knowledge is generally acknowledged, by both design and service engineers, as a rich resource to the company. There is also a general consensus is that it should be exploited more than it is currently the case.

\section{Methods of codification}

It is envisaged that a PSS business with extended lifecycle will need to make much greater use of codification approaches to enhance its activities compared with traditional business model. As the requirement for extended support may be required for 30 years and beyond for complex systems such as aircrafts, issues such as personnel turnover and distributed teams and supply chain mean that codification approaches are necessary. Focusing on codification approaches, we report our experiences associated with in-service information capture and feedback and suggest methods to enhance opportunities for reusing in-service information collection.

\subsection{In-service information capture}

In traditional product offering businesses, operational and in-service information is often collected and owned by the customers and not the designing company. In this situation, the sharing and feedback of service information is limited and depended greatly on the contractual and commercial interests of the organisations involved. Information about the performance and failures is often sensitive and contentious as it was pointed out on several occasions that customers may not wish to share such information, for example when this might affect their interests. 
The move towards through-life management within the PSS should allow for higher levels of data sharing between customers, manufacturers and service providers. Contracts based on availability targets need detailed reliability, failure and usage data to be shared (Ward and Graves 2006). The data on performance and use can now be collected by the designing companies with diagnostics and prognostics technologies integrated. A number of authors have used the term "informated products" to refer to these (Neely 2007). Rolls-Royce TotalCare ${ }^{\circledR}$ for instance, offers a suite of engine support services including; proactive in-service engine management, overhaul shop maintenance and the collection and management of engine data. The crucial differences in the PSS arrangements is the feedback is not through second or third party maintenance centre or operators but by the designing companies. Therefore, the company can control what information is captured, without the complication of commercial sensitivities. This possibility to collect operational data offers new opportunities for improvement that wasn't readily available in traditional businesses.

It is important to recognized that a wide range of codifications methods used to capture information from service and use but most recent literature on this subject has focused on the data logging and monitoring systems (Jardine et al. 2006). Our literature survey reveals that monitoring systems are used mainly to enhance maintenance activities (e.g predictive maintenance) where decision-support algorithms are developed. As an example, the Distributed Aircraft Maintenance Environment (DAME) is an e-Science project demonstrating the use of GRID infrastructure to implement online health monitoring and fault diagnostic systems for the maintenance of aircraft engines in distributed environments (Ong 2004). Examples of other approaches include online customer and service reporting systems, maintenance and service records and reports etc. Most reporting systems generate records from standard forms, or based on templates, and can be uniquely identified (by serial number) to allow each instance to be referenced and traced. Increasingly common practice in aerospace industry, non-trivial experiences may also be recorded in best practice and lessons learned databases for future reference (Lowe et al. 2003). The aim is mainly to support sharing and distribution of knowledge (in the form of lessons learned) to the wider organisation in order to avoid repeating the same mistakes. Lessons learned system is usually generic and is not limited to supporting in-service feedback but also other activities within the organisation. These systems rely on contribution by critical employees and face with motivational and cultural challenges. The challenges we observed from an aerospace company are also reported by NASA, which has one of the most comprehensive lessons learned information system (NASA 2007).

The codification techniques result in wide ranging content and representations of in-service information such as databases and text-based documents and will require different approaches in reuse. In the case of database, the structure itself can be exploited for reuse but ensuring data quality is a critical issue. There must be guidelines and a verification process in place to ensure the data is descriptive of the underlying event being reported. Similarly in text records, the importance of a non-discriminating ${ }^{1}$ reporting mechanism is particularly highlighted, especially in the reporting of lessons-learned instances. This practice needs to be encouraged throughout the organisation to obtain the actual depiction of in-service experiences so that improvements can be prioritised effectively.

It is apparent that the limitation in information reuse is due to the lack of upfront strategies associated with the information capture. For instance, in the OREDA experience (Sandtorv et al. 1996), it was reported that the planned use of data must be identified and specified before collection, and "nice to have" data collection should be avoided. Therefore, the purpose and targeted audience need to be known at the point of information capture. In one company, the data logging capability only serves as a sales feature to compete with the market leader therefore there is no active role in collecting and analysing the data. In another, maintenance records are captured by service personnel without the intention of reuse at all (as an event log for invoicing the customer). These practices result in poor and incomplete records that are difficult to manage and organise. We

\footnotetext{
${ }^{1}$ Instances should be objectively reported without being subjected to prejudices.
} 
believe the PSS companies could take greater benefits of reuse if strategies for knowledge and information management are considered from the outset when implementing the information systems.

\subsection{In-service information reuse}

Information reuse occurs when information is assimilated and used in a new application and also, subject to some processing, yields useful new insights and knowledge. In developing approaches to facilitate reuse of inservice records in an aerospace company, our experience shows that greater use of structured records ${ }^{2}$, controlled vocabulary and taxonomy is fundamental. By analysing a set of in-service records collected by the company (mixture of numerical, controlled and free-text descriptions), we found that errors, inconsistent use of terminology, abbreviations etc. are still common despite guidelines being given to consider potential retrieval requirements at the point of capture. However, many concepts were repeatedly referred to but the same concepts may not be immediately identifiable from the records. Hence, the pre-processing efforts required (terms extraction and thesauri construction) are not trivial requiring laborious manual efforts and rationalisation by the domain experts (dealing with spelling variations, synonyms, acronyms etc.). Although a wealth of information is being captured and stored, the information may be held in various representations and is not available readily to the right people in the right time. In many cases, engineers have to request for additional information in order to perform their tasks and also to assimilate information from numerous disparate records. Indifferent to other large or medium-sized companies, the tools and databases usually evolve independently within departments that create and maintain them. This means the databases are scattered, inconsistent and difficult to share and coordinate even amongst teams involved in the same activities, in this case, between in-service support teams dealing with different components of the aircraft. Efforts for harmonising the databases and improving the records are under development but at present, those issues hinder the reuse process making it tedious and less amenable to knowledge discovery.

A number of techniques can be employed to analyse and reuse data or information collected from in-service to make useful inferences (therefore to facilitate knowledge discovery) if information is structured and with much better use of controlled vocabulary (or taxonomy) within the structure. We discuss two key classes of techniques that are useful for knowledge discovery. The first approach is based on information classification, which is the approach we have adopted in organising the in-service records of the aerospace company; the second approach is using statistical analysis and data mining techniques, which are considered useful to automate the knowledge discovery process.

\subsubsection{Information classification}

Classification hierarchies, concept hierarchies and groups of subject headings are all examples of taxonomies and are used synonymously to describe structures used to organise information. Greater structure and controlled vocabulary used in the in-service records will help towards information organisation using taxonomy, or classification. Taxonomic information organisation is useful for retrieval, as an alternative to query-based retrieval. In particular, a faceted classification approach (Rowley and Farrow 2000), which can be viewed as employing multiple concurrent classification schemes may be a useful approach in organising service records. In a faceted scheme, facets are "clearly defined, mutually exclusive, and collectively exhaustive aspects, properties or characteristics of a class or specific subject" (Taylor, 1992). For the inservice records, these can be the operator, location, date, faulty part, cause, failure modes etc. By organising in-service records into a faceted classification, enhanced browsing is supported and it becomes possible to discern correlation and trends between facets of interest (see Figure 1 (a)). The implementation of the faceted

\footnotetext{
2 'Structured record' is that which is represented electronically in a way that makes it interpretable to a computer, such that a computer can understand what to do with it when it is encountered.
} 
classification is through the Waypoint system (McMahon et al. 2002), the full development of the system is being reported elsewhere (Goh et al. 2008).

\section{Figure 1 Faceted classification of in-service records (a) classification tree (b) list of results based on concepts selected.}

At the point of retrieval, the users may select a combination of concepts to arrive at a much reduced records that are highly relevant (only records that satisfy the conjunction of the selected concepts) to expedite retrieval and help decision-making with the in-service issues hence their responses to customers. For example, by choosing the Aircraft Type "XYZ" the users reduce the records to exclude all other types of aircrafts. This is done by ticking the box next to the concept node in the classification tree shown in Figure 1 (a). All records that are not relevant to "XYZ" will be pruned from the tree (McMahon et al. 2002). The users can introduce an additional constraint by selecting the Assembly "Main Landing Gear" to further reduce the records. This is again done by ticking the box next to the concept node labelled "Main Landing Gear" shown in the same figure. The more constraints are used the remaining/retrieved records become smaller and more relevant to the queries.

Additionally, by browsing through the faceted schemes, engineers might be able to discover classes of inservice issues that are recurrent/systemic such as design-induced (if there is apparent correlation between a component/topological location and a failure mode), operation-induced (if there is apparent correlation between an operational phase/event and a failure mode) and use-induced (if there is apparent correlation between a flight type/route/aircraft operator and a failure mode) issues. Correlation can be indicated by higher than average number of instances between two or more concept nodes. Although the correlations do not necessarily indicate an underlying problem, by indicating that it is a frequent occurrence, it acts as a prompt for engineers to determine root causes, which may lead to understanding of the operating conditions practiced by different airlines (e.g. maintenance procedure, type of operation - short or long haul). As an example, given the classification of in-service events according to "Operator" and "Fault Type" facets, we may be able to discover the occurrence of a specific fault type that is more likely to occur with a specific operator from the intersection of these two facets. This discovery may allow engineers to infer the operating regimes, for instance, which may not have been readily apparent to the designing company. This way, the faceted classification schemes help to realise the double-loop learning mode that may lead to designing out certain aspects of the product. Methods for supporting this mode of learning, as previously state, have been limited so far.

\subsubsection{Statistical analysis and data mining}

One of the key tasks in classifying the in-service records involved structuring to make the records' content semantically meaningful. For instance, the term 'landing' may refer to the Operational Phase "Landing" or the Assembly "Landing Gear" in the in-service records. In the case study, the records were scripted into XML documents with its contents tagged with standard headings. With a collection of structured records, methods usually employed in statistical analysis and data mining (knowledge discovery) can now be exploited to discover patterns or correlations in the data that may not be previously apparent. Data mining involves a series of stages including business understanding, data understanding, data preparation, modelling, evaluation and deployment according to the CRISP-DM process model (http://www.crisp-dm.org/Process/index.htm). A number of algorithms and visualisation techniques are available and can be readily applied to reveal, interrogate data in different ways to arrive at useful, and often, hidden models or patterns. Statistical analyses are also employed in a data mining process. In reliability of electronic products, James et al. (James et al. 2002) explained the use of "slice and dice" analysis to enable the analyst to look at root cause and trend data 
across specific criteria such as product type, lifecycle episodes e.g. particular manufacturing batch points), application, or individual user. This may give the analyst further understanding of the nature of the reported events that may be characteristic of the environment in which the artefact is operating. By focusing on understanding such correlation, knowledge to enhance design decisions aimed at mitigating root causes of inservice issues can be actively acquired.

Structuring and organising the in-service records alone provide great potential for learning through knowledge discovery as described. Nevertheless, for greater reuse especially to relate in-service issues to design and manufacturing factors (i.e. resembling the double-loop learning mode), methods for linking or crossreferencing the in-service instances to design or manufacturing records will be necessary. If this can be achieved it may allow for flaws related to the design features or methods, or the manufacturing batches or conditions to be evident through analyses. For example, instead of reporting a generic component such as 'safety valve' the part numbers and serial numbers can be used to trace faulty parts through the development process. From the case study experience, we conclude that the information captured about the in-service events can be further improved to include taxonomical descriptions of the issues and to develop crossreferencing between information held on different systems and repositories. The cross-referencing or mapping between systems in particular will enable richer information sets to be linked and analysed. At present, this latter task has yet to be tested but it is foreseen to involve significant intellectual efforts.

\section{Conclusions}

To this end, feedback from service represents a significant resource for learning in companies embracing the PSS business model and allows enhanced competitiveness through capitalisation of this resource. For instance, the knowledge gained about the functional or operational performance of the product through its use can provide a basis for assessing and updating design knowledge thus designing more reliable and serviceable products. At present, service observation is fed back through unstructured and disparate records making it difficult to collate and analyse. Therefore, learning-from-use relies predominantly on laborious manual trawling through information or on personalisation approaches. Inevitably for the PSS companies to continually learn from use will involve a series of organisation strategies, processes, tools and methods to capture and reuse the data and information to acquire knowledge. We believe that reuse of information collection should be considered from the outset such that its content and representation could be enhanced and made more amenable to computational analysis and organisation for knowledge discovery. This is a major drawback observed in many organisations.

In conclusion, we summarise four strategies where we see emphasis can be given to promote success of codification approaches.

- Incentivise the people to capture information effectively. The issue of incentives emerged in many interactions we had with the engineers and is also apparent in many references, for example NASA (2007). This is particularly important when the codification method will require contribution from people such as the lessons learned system. Given pressure to deliver on task or project, capturing lessons learned is often not viewed as a priority by engineers. One of the main reasons for lack of interest in contributing to information capturing is due to the lack of understanding of the purpose or reuse benefits. Providing incentives may alleviate some of these resistances until benefits from reuse become evident. In addition, champions and support teams can be engaged to undertake initiatives and promote awareness, train and encourage buy-in.

- Points of capture and reuse must be embedded within work processes to ensure effectiveness. Information capture and reuse is triggered by the need to carry out a task. Due to similar reasons stated above, people often develop workarounds and shortcuts when under strict deadlines. Therefore information capture should be automated where possible to reduce work loads such as through use of templates, assisted 
systems etc. Most importantly, reuse must be considered as early as possible where the impacts will be the greatest. One such effort in the company we observed was to incorporate such consideration during initial review meetings.

- Adoption of formal taxonomies (or controlled vocabulary) and standardised information structures and representation to improve reuse. Despite a wealth of information being captured from service, information reuse is often limited due to inconsistency in the representation and structure. We believe opportunities for reuse to improve the maintenance operations as well as the design capabilities of the PSS companies can be enhanced with efforts to develop and standardise taxonomies, vocabulary, information structure and representation (at least within domains/work groups) as well as training engineers to capture information more effectively.

- Improved retrieval and organisation of information to add value to intellectual asset. Information that is not retrievable is lost and cannot be reused. Methods for improving retrieval and organisation of information are critical to ensure information is available to the right people at the right time. Contextbased reuse may be efficient but its effectiveness cannot be guaranteed or time-proof. It may also limit collective reuse because the crucial pieces of information may not have been captured. Hence, investment into such activities will add value to the intellectual asset through knowledge discovery.

It is in the authors' contention that the benefits from reuse may then provide some means towards incentivisation for information capture in the first place.

\section{Acknowledgements}

The authors gratefully acknowledge the funding provided by the Engineering and Physical Science Research Council (EPSRC) for the KIM Project (http://www-edc.eng.cam.ac.uk/kim/) under Grant No. EP/C534220/1 and the IdMRC under Grant No. GR/R67507/01 for the research reported in this paper. The contribution of industrial collaborators of the KIM project is also gratefully acknowledged.

\section{References}

Alonso-Rasgado, T., Thompson, G. and Elfström, B.-O., 2004. The design of functional (total care) products. Journal of Engineering Design, 15 (6), 515-540.

Argyris, C., 1999. On organizational learning, 2nd Ed. ed. Oxford: Blackwell.

Busby, L.S., 1998. The neglect of feedback in engineering design organisations. Design Studies, 19, 103-117.

Doultsinou, N., Roy, R., Baxter, D. and Gao, J., 2007. Identification of service knowledge types for technical product-service systems. 4th International Conference on Digital Enterprise Technology, DET 2007. Bath, UK, 549-555.

Fundin, A.P., Bergman, B. L. S., 2003. Exploring the customer feedback process. Measuring Business Excellence, 7 (2), 56-65.

Goh, Y.M., Giess, M.D., Stewart, D. and McMahon, C.A., 2008. Application of faceted classification to inservice records. Proc. IDMME 2008 Virtual Concept. Beijing, China, under review.

Hansen, M.T., Nohria, N. and Tierney, T., 1999. What's your strategy for managing knowledge? Harvard Business Review, 77 (2).

Jagtap, S., Johnson, A., Aurisicchio, M. and Wallace, K., 2007. In-service information required by engineering designers. ICED'07. Paris, France.

James, I.J., Marshall, J. and Walls, L., 2002. Improving design for reliability with in-service data analysis. Proc. Annual Reliability and Maintainability Symposium. 182. 
Jardine, A.K.S., Lin, D. and Banjevic, D., 2006. A review on machinery diagnostics and prognostics implementing condition-based maintenance. Mechanical Systems and Signal Processing, 20, 14831510.

Koudal, P., Lee, H. L., Whang, S., Peleg, B., Rajwat, P., 2004. OnStar: Connecting to customers through telematics. Global Supply Chain Management Forum.

Lowe, A., McMahon, C.A., Culley, S.J., Coleman, P. and Dotter, M., 2003. A novel approach towards design information management within airbus. ICED'03. Stockholm, Sweden.

Maidique, M.A., Zirger, B. J., 1985. The new product learning cycle. Research Policy, 14 (6), 299-313.

Márquez, A.C. and Herguedas, A.S.N., 2004. Learning about failure root causes through maintenance records analysis. Journal of Quality in Maintenance Engineering, 10 (4), 254-262.

McMahon, C., Crossland, R., Lowe, A., Shah, T., Williams, J.S. and Culley, S., 2002. No zero match browsing of hierarchically categorized information entities. Artificial Intelligence for Engineering Design, Analysis and Manufacturing, 16 (243-257).

NASA, 2007. The NASA engineering network [online]. http://www.nasa.gov/offices/oce/llis/home/index.html [Accessed Access Date 2007].

Neely, A., 2007. The servitization of manufacturing: An analysis of global trends. Proc. of the POMS College of Service Operations and EurOMA Conference. London.

Ong, M., Ren, X., Allan, G., Kadirkamanathan, V., Thompson, H. A., Fleming, P. J., 2004. Decision support system on the grid. Int'l Conference on Knowledge-Based Intelligent Information \& Engineering Systems. New Zealand.

Rowley, J. and Farrow, J., 2000. Organising knowledge, 3rd ed. Aldershot, Hants, UK: Gower.

Sander, P.C. and Brombacher, A.C., 2000. Analysis of quality information flows in the product creation process of high-volume consumer products. International Journal of Production Economics, 67 (1), 37-52.

Sandtorv, H.A., Hokstad, P. and Thompson, D.W., 1996. Practical experience with a data collection project: The OREDA project. Reliability Engineering \& System Safety, 51 (2), 159-167.

Tan, A.R. and McAloone, T.C., 2006. Characteristics of strategies in product/service-system development. DESIGN 2006. Dubrovnik, Croatia.

Ward, Y. and Graves, A., 2006. Through-life management: A catalyst for process excellence in customer support and maintenance, repair \& overhaul (MRO). Bath, UK.

Wenger, E., 2000. Communities of practice and social learning systems. Organization, 7 (2), 225-246.

Wong, S.C., Crowder, R.M., Wills, G.B. and Shadbolt, N.R., 2007. Informing preliminary design by incorporating service knowledge. International Conference on Engineering Design. Paris, France. 


\section{Figure Captions}

Figure 1 Faceted classification of in-service records (a) classification tree (b) list of results based on concepts selected.

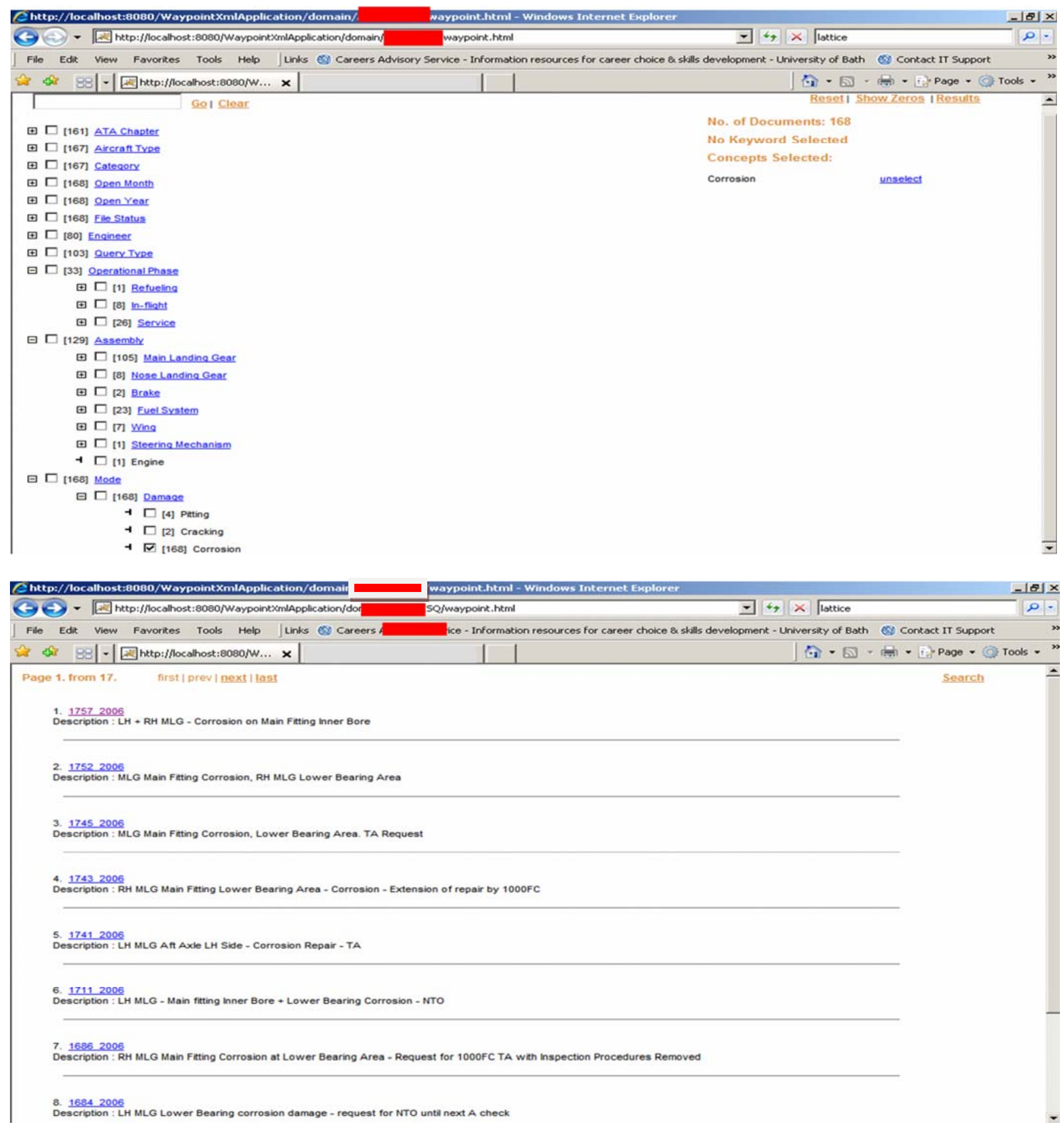

